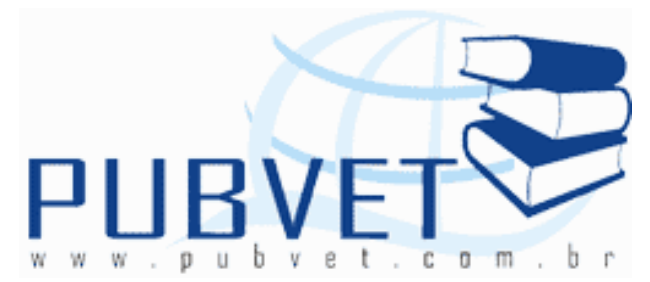

PUBVET, Publicações em Medicina Veterinária e Zootecnia.

\title{
Avaliação da eficiência de resíduos agroindustriais como fonte de nutrientes para pastagens
}

Mérik Rocha Silva ${ }^{1}$, Camila Souza Magela de Menezes ${ }^{1}$, Diogo Francisco

Peixoto de Oliveira ${ }^{1}$, Thiago Alves Reis ${ }^{1}$, Uanderson Veríssimo Luna ${ }^{1}$, Maria Aparecida Pereira Pierangelli ${ }^{2}$

${ }^{1}$ Bacharéis em Zootecnia - UNEMAT. e-mail: merikrocha@hotmail.com

${ }^{2}$ Prof. do Departamento de Zootecnia - UNEMAT. e-mail: mapp@unemat.br

\section{Resumo}

Este trabalho foi realizado com o intuito de avaliar o potencial de utilização dos principais subprodutos das agroindústrias da região do Vale do Guaporé (Pontes e Lacerda, MT) na adubação de pastagens de Brachiária híbrida (cruzamento de $B$. brizantha, $B$. ruziziensis e $B$. decumbens). Soro de leite bovino e resíduo ruminal produzidos nas agroindústrias locais foram aplicados como adubos orgânicos, em quantidades para disponibilizar 0, 50, 100 e $200 \mathrm{~kg}$ de $\mathrm{N}$ por ha. Além das fontes orgânicas, o experimento constituiu de uma adubação convencional e um tratamento testemunha. $O$ resíduo ruminal e o soro de leite bovino foram incorporados nos solos de cada vaso, separadamente, 15 dias antes do plantio. Os melhores resultados foram apresentados pelas forrageiras adubadas com soro de leite bovino, entre os tratamentos com utilização de resíduo ruminal, destacou-se o tratamento com 25 toneladas de resíduo ruminal por ha.

Palavras-chave: adubação de pastagens, resíduos frigoríficos, resíduos laticínio. 


\title{
Evaluation of the efficiency of agro-industrial waste as a source of nutrients for pastures
}

\begin{abstract}
This work was carried out in order to evaluate the potential for use of the main products of agricultural industries in the region of Vale do Guaporé (Pontes e Lacerda, MT-Brazil) in the fertilization of pastures of Brachiária water (crossing of $B$. brizantha, $B$. ruziziensis and $B$. decumbens). The bovine whey, and waste produced in the rumen agroindustries sites were used as organic fertilizers, aiming to optimize the production of natural pastures intended for ruminant animals. -Seeking to provide $0,50,100$ and $200 \mathrm{~kg} \mathrm{~N}$ per hectare (ha). The residue produced in the rumen of the refrigerator and bovine whey, were separately incorporated in the soil 15 days before planting, fodder compared the results with each other and with conventional fertilizer (chemical fertilization of macroelements-NPK) beyond the results of production without fertilizers or corrective.
\end{abstract}

Keywords: dairy waste, fertilization of pastures, slaughterhouse waste

\section{Introdução}

O Brasil é beneficiado por vastas áreas de solos férteis, no entanto têm manejado mal a maioria dos seus solos, especialmente as áreas onde são cultivados suas pastagens que lhe dão condições de ser o maior exportador mundial de carne bovina (ANUALPEC, 2008). Esta falta de atenção para com os solos resulta na redução do potencial produtivo das pastagens, decorrentes da acidez e da ineficiente ou inexistente reposição dos nutrientes, refletindo em pastagens deficientes em nutrientes e minerais. Isso compromete o potencial produtivo dos animais que consomem esta forragem (Júnior, Vilela \& Souza, 2007).

O valor econômico das pastagens tem sido elevado paulatinamente, devido aos resultados produtivos da pecuária que estão comprometidos as condições das pastagens. Inovações dos meios de produção empregados na 
bovinocultura brasileira deverão objetivar a sustentabilidade da criação de animais a pasto no país. Para isso é necessário adotar métodos inovadores e eficientes de renovação da fertilidade do solo, incluindo no processo de produção a destinação segura dos resíduos, bem como seu reaproveitamento. A degradação das pastagens na região dos Cerrados potencializa um impacto negativo sobre os recursos hídricos da nação, devido seis das oito grandes bacias hidrográficas brasileiras terem suas nascentes no Cerrado (Júnior, Vilela \& Souza, 2007).

É indicado a utilização de adubos alternativos, que apresentem custos viáveis e oferta suficiente. A inovação no processo de produção pecuária utilizando resíduos do próprio sistema para elevar o potencial produtivo das pastagens, repercute positivamente, promovendo a sustentabilidade e a criação de uma nova fonte de nutrientes independente dos onerosos sistemas de extração mineral.

\section{Material e Métodos}

O experimento foi realizado em casa de vegetação localizada nas dependências do Campus Universitário de UNEMAT em Pontes e Lacerda,MT. Foram utilizados 36 vasos plásticos preenchidos com $6 \mathrm{~kg}$, cujas caracterização da fertilidade é mostrada na tabela 1.

Realizamos dois grupos de quatro tratamentos com quatro repetições, utilizando separadamente o soro de leite bovino e o resíduo ruminal bovino para disponibilizar $0,50,100$ e $200 \mathrm{~kg}$ de $\mathrm{N}$ por ha. No caso do resíduo ruminal foi estimado conforme Ribeiro (1999) que 33\% do $\mathrm{N}$ analisado seria disponibilizado ao vegetal neste primeiro cultivo, estabelecendo dosagens conforme tabela 2. Paralelamente, outro tratamento com adubação química com quatro repetições alcançando $50 \mathrm{~kg}$ de $\mathrm{N}$ por ha foi conduzido no intuito de obter dados referentes à produção de matéria natural no método convencional de adubação. A aplicação do soro de leite bovino foi realizada por meio de 
SILVA, M.R. et al. Avaliação da eficiência de resíduos agroindustriais como fonte de nutrientes para pastagens. PUBVET, Londrina, V. 5, N. 17, Ed. 164, Art. 1111, 2011.

irrigação e o resíduo ruminal foi totalmente fragmentado e totalmente incorporado ao solo.

Tabela 1: Caracterização química do solo, resíduo ruminal e soro de leite bovino utilizados.

\begin{tabular}{l|c|c|c}
\hline & SOLO & $\begin{array}{c}\text { RESÍDUO } \\
\text { RUMINAL com } \\
60 \% \text { umidade }\end{array}$ & $\begin{array}{c}\text { SORO DE LEITE } \\
\text { BOVINO }\end{array}$ \\
\hline $\mathrm{pH}$ em água & 6,0 & 6.95 & $\mathrm{xxx}$ \\
$\mathrm{pH}$ em CaCl & 6,10 & $\mathrm{xxx}$ \\
Matéria Orgânica & 5,1 & $\mathrm{xxx}$ & $\mathrm{xxx}$ \\
$(\mathrm{dag} / \mathrm{kg})$ & 1,2 & 181,6 & $\mathrm{xxx}$ \\
$\mathrm{K}\left(\mathrm{mg} / \mathrm{dm}^{3}\right)$ & 121,3 & 0,7 & $\mathrm{xxx}$ \\
$\mathrm{Ca}^{2+}\left(\mathrm{cmolc}^{3} \mathrm{dm}^{3}\right)$ & 1,3 & 0,4 & $\mathrm{xxx}$ \\
$\mathrm{Mg}^{2+}\left(\mathrm{cmolc}^{3} \mathrm{dm}^{3}\right)$ & 0,8 & 0 & $\mathrm{xxx}$ \\
$\mathrm{Al}^{3+}\left(\mathrm{cmolc} / \mathrm{dm}^{3}\right)$ & 0 & 1,3 & $\mathrm{xxx}$ \\
$\mathrm{H}+\mathrm{Al}\left(\mathrm{cmolc} / \mathrm{dm}^{3}\right)$ & 2,3 & $\mathrm{xxx}$ & $\mathrm{xxx}$ \\
$\mathrm{CTC} \mathrm{Efetiva}$ & 2,4 & 12 & $* 1,3 \mathrm{~g} / \mathrm{L}$ \\
$\left(\mathrm{cmolc} / \mathrm{dm}^{3}\right)$ & & $\mathrm{xxx}$ & $* 1,1 \mathrm{~g} / \mathrm{L}$ \\
$\mathrm{N}(\mathrm{g} / \mathrm{Kg})$ & $\mathrm{xxx}$ & $\mathrm{xxx}$ & $* 1,9 \mathrm{~g} / \mathrm{L}$ \\
$\mathrm{P}_{2} \mathrm{O}(\mathrm{g} / \mathrm{L})$ & $\mathrm{Xxx}$ & & \\
$\mathrm{K}_{2} \mathrm{O}(\mathrm{g} / \mathrm{L})$ & \multicolumn{2}{c}{} \\
\hline
\end{tabular}

*Adapatado de GHERI, 2003.

Tabela 2: Tratamentos

\begin{tabular}{l|c}
\hline \multicolumn{1}{c|}{ TRATAMENTOS } & NÚMERO DOS VASOS \\
\hline Sem resíduo ruminal & $1,5,9$ e 13 \\
$37,5 \mathrm{~g}$ por vaso (50kg de N/ha) & $2,6,10$ e 14 \\
$75 \mathrm{~g}$ por vaso (100kg de N/ha) & $3,7,11$ e 15 \\
$150 \mathrm{~g}$ por vaso (200kg de N/ha) & $4,8,12$ e 16 \\
Sem soro de leite bovino & $17,21,25$ e 29 \\
$350 \mathrm{ml}$ por vaso (50kg de N/ha) & $18,22,26$ e 30 \\
$700 \mathrm{ml}$ por vaso (100kg de N/ha) & $19,23,27$ e 31 \\
$1400 \mathrm{ml}$ por vaso (200kg de N/ha) & $20,24,28$ e 32 \\
Adubação química comum - NPK (50kg de N por ha) & $33,34,35$ e 36 \\
\hline
\end{tabular}

Os resíduos permaneceram quinze dias incorporados no solo com intuito de condicionar meios de haver a disponibilização dos nutrientes as plantas. Posteriormente foi realizado novo revolvimento dos solos a fim de 
SILVA, M.R. et al. Avaliação da eficiência de resíduos agroindustriais como fonte de nutrientes para pastagens. PUBVET, Londrina, V. 5, N. 17, Ed. 164, Art. 1111, 2011.

homogeneizar o máximo possível o material. Em seguida foram plantadas cinco sementes peletizadas de Brachiária Mulato $\mathrm{II}^{\circledR}$ por vaso.

Os vasos forram irrigados diariamente, evitando saturar o solo com água e não comprometer o metabolismo dos vegetais pela deficiência de $\mathrm{H}_{2} \mathrm{O}$. O corte das plantas, a cinco $\mathrm{cm}$ do solo, foi realizado sessenta dias após o plantio, respeitando o período mínimo de estabelecimento das gramíneas conforme orientação do fornecedor. Em seguida os materiais foram pesados para obtenção da produção de biomassa verde (BV).

O resíduo ruminal é o conteúdo do rúmen, omaso e retículo bovino retirado do animal abatido em frigorífico. Salientamos que este resíduo foi degradado em quase toda sua totalidade pela microflora ruminal, no entanto não chegou a passar pelos intestinos, onde os nutrientes em sua maioria são absorvidos, o que resulta em subproduto de maior qualidade que o resido ruminal excretado (fezes). Foi avaliado a realização da adubação de pastagens com os mencionados resíduos, visando estabelecer uma forma eficiente de impedir problemas ambientais com o volume de resíduos de forma economicamente sustentável. Analisamos os resultados produtivos das forrageiras adubadas com os resíduos agroindustriais supracitados.

\section{Resultados e Discussão}

É de amplo conhecimento a importância da matéria orgânica na qualidade física e química do solo. Ela atua na elevação da CTC (capacidade de troca de cátions), potencializa a retenção de água nos micro e macro poros, interfere positivamente na formação de agregados e melhora as atividades biológicas (KLUTHCOUSKI et al, 2003). A matéria orgânica, através da decomposição torna-se húmus, que é adubo altamente eficiente contribuindo para conservação da água e do solo. Todavia, um adubo eficiente deve oferecer nutrientes suficientemente eficazes de elevar a produtividade das plantas. Além de todas essas características a oferta do adubo a ser utilizado deve ser quantitativamente e economicamente acessível. Em contraste com o alto custo 
SILVA, M.R. et al. Avaliação da eficiência de resíduos agroindustriais como fonte de nutrientes para pastagens. PUBVET, Londrina, V. 5, N. 17, Ed. 164, Art. 1111, 2011.

de adubos, surge a necessidade de reduzir custos, e evitar impactos ambientalmente negativos da atividade agropecuária no meio ambiente. Isso pode ser conseguido com a utilização de resíduos orgânicos provenientes de diversas fontes, entre elas, as agroindústrias, tais como frigoríficos e laticínios.

A bovinocultura leiteira altamente produtiva produz como resíduo industrial o soro de leite, que é na maioria dos casos disponibilizados gratuitamente ao agricultor. Segundo Antunes (2003) as proteínas do soro variam de acordo com a fonte do leite, método de preparação e tipo de queijo produzido e especificações individuais dos processadores; o que com certeza repercutira o percentual de $\mathrm{N}$ e a disponibilização deste aos vegetais presentes no solo (taxa de biodegradabilidade).

Ocorreu aumento de produção de BV nos vasos que receberam tratamentos com 116 e 232 mil litros de soro por ha (Figura 1); e as forrageiras cultivados em solo adubados com 464 mil litros de soro de leite bovino por ha apresentaram a menor produção, sendo superados em produção inclusive pelos vasos que não receberam adubação. 465 mil litros de soro por hectare aplicados antes do sementeio apresentaram resultados extremamente desinteressantes. Pressupõe-se que todos nutrientes presentes no soro já estavam disponíveis aos vegetais durante a germinação. No entanto no resíduo ruminal como esperado sofreu biodegradação lenta, compreendendo que este solo terá nutrientes a serem disponibilizados ao vegetal posteriormente.

\section{Conclusões}

A utilização de resíduos agroindustriais na adubação de pastagens em formação demonstrou resultados promissores. A utilização do soro de leite bovino disponibiliza nutrientes que repercutem uma produção de biomassa quase quatro vezes maior que adubação química através do tratamento com 116 mil litros por ha; disponibilizando em torno de $50 \mathrm{~kg}$ de $\mathrm{N} / \mathrm{ha}$. A partir desta quantidade o soro de leite bovino demonstrou ser eficaz na adubação, mas com resultados inferiores ao tratamento com 232 mil litros/ha 
SILVA, M.R. et al. Avaliação da eficiência de resíduos agroindustriais como fonte de nutrientes para pastagens. PUBVET, Londrina, V. 5, N. 17, Ed. 164, Art. 1111, 2011.

O resíduo ruminal apresentou bons resultados, comparados a adubação convencional, sendo melhor a produção obtida com aplicação de 25 toneladas por ha. O resíduo ruminal apresentou resultados produtivos durante os dois meses de cultivo dos vegetais, mas, inferior aos resultados com aplicação de soro.

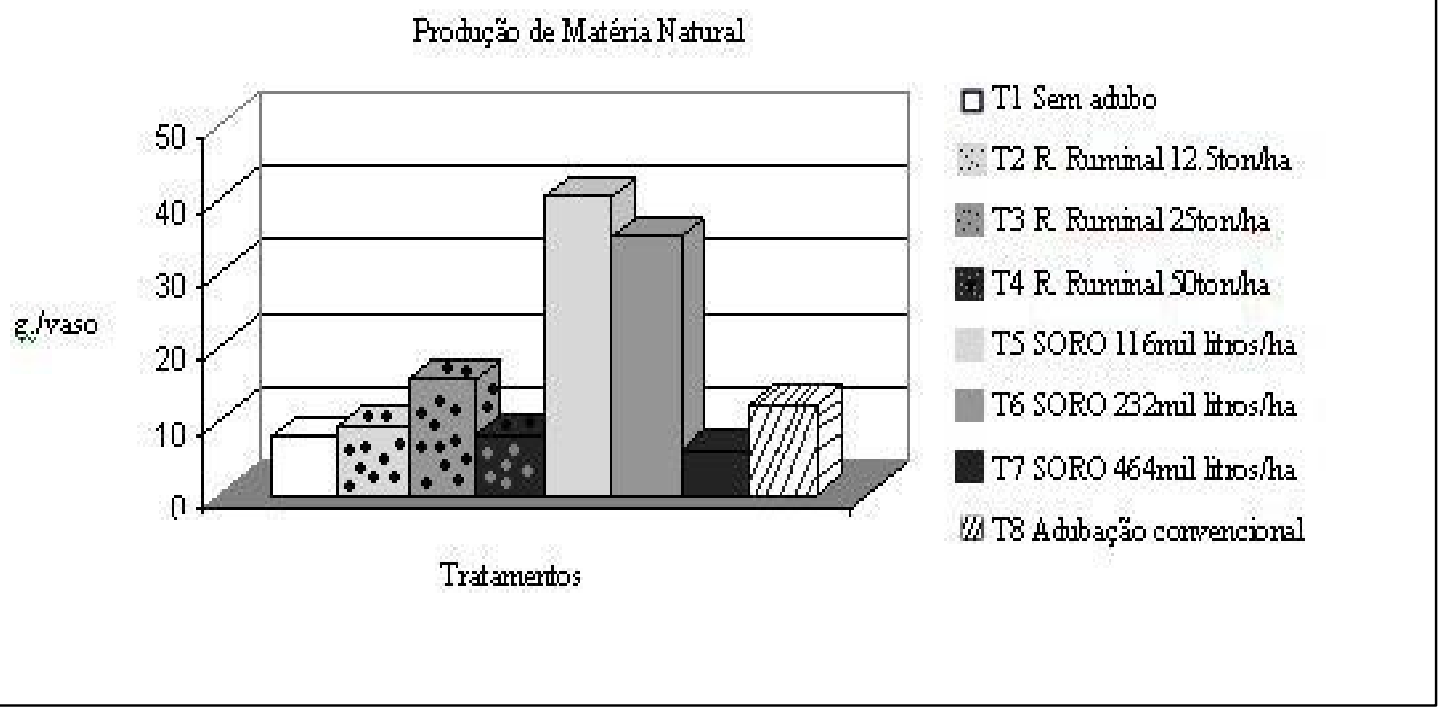

Figura 1: Gráfico de produção de matéria natural.

\section{Literatura citada}

ANUALPEC 2008 Anuário da Pecuária Brasileira. São Paulo: Instituto FNP, 2008. 380 p.

ANTUNES, A.J. Funcionalidade de Proteínas do Soro de Leite Bovino - Barueri, SP: Manole, 2003. 127 p.

GHERI. E.O., FERREIRA, M.E. e CRUZ, M.C.P. Resposta do capim-tanzânia a aplicação de soro acido de leite. Brasília, DF: Pesquisa Agropecuária Brasileira, 2003. 760 p.

JÚNIOR, G.B.M. VILELA, L. DE SOUZA, D.M.G. CERRADo Uso Eficiente de Corretivos e Fertilizantes em Pastagens. Planaltina, DF: Embrapa Cerrados, 2007. 224 p.

KLUTHCOUSKI, J. STONE, L.F. AIDAR, H. Integração Lavoura-pecuária. Santo Antônio de Goiás: Embrapa Arroz e Feijão, 2003. 570 p.

RIBEIRO, A.C. et. al. Recomendações para Uso de Corretivos e Fertilizantes em Minas Gerais $5^{a}$ aproximação. Viçosa: Independente, 1999. 359 p. 